\title{
Report
}

\section{Glaucoma and Congenital Zika Syndrome}

Ocular lesions are a prominent feature of congenital Zika virus (ZIKV) infection, ${ }^{1-3}$ in addition to microcephaly and severe central nervous system defects. ${ }^{4}$ Such manifestations, which include chorioretinal atrophy, focal pigmented mottling, and optic nerve abnormalities, have been for the most part restricted to the posterior segment. ${ }^{1-3}$ Glaucoma is a rare sequel of congenital infections and at present, has not been described among infants exposed to ZIKV during gestation.

Herein, we report a 3-month-old male infant who was born during the microcephaly outbreak in the city of Salvador, Brazil, and presented with an enlarged right eye, photophobia, and persistent tearing. During the fourth week of pregnancy, the mother had an acute illness characterized by cutaneous rash, fever, and arthralgia that lasted 3 days. An infant weighing $1.892 \mathrm{~kg}$ was delivered by caesarian section at 38 weeks of gestation who had severe microcephaly $(-4.54$ standard deviation below the InterGrowth standard), bilateral lower extremity arthrogryposis, and according to cranial computed tomography, ventriculomegaly, diffuse parenchymal calcifications, dysgenesis of the corpus callosum, and a simplified gyral pattern. An ophthalmologic evaluation, performed 3 days after birth, found chorioretinal atrophy and focal pigmented mottling in both eyes and optic nerve hypoplasia in the right eye, signs consistent with glaucoma were not identified. Sera obtained at birth tested positive for anti-ZIKV immunoglobulin $\mathrm{M}$ antibodies and negative for anti-dengue virus immunoglobulin $\mathrm{M}$ antibodies, as well as for other causes of congenital infection. Real-time reverse transcriptase polymerase chain reaction testing of newborn blood did not detect ZIKV RNA.

During an outpatient visit 95 days after birth, the infant was found to have an enlarged right eye and persistent tearing. The mother did not report additional ocular symptoms before the evaluation. The infant displayed significant irritability and severe photophobia. The right eye had an increased horizontal corneal diameter in comparison to the left eye $(13 \mathrm{~mm}$ in the right eye vs $10 \mathrm{~mm}$ in the left eye; Fig 1) and an increased intraocular pressure (30 $\mathrm{mm} \mathrm{Hg}$ in the right eye vs $14 \mathrm{~mm} \mathrm{Hg}$ in the left eye). The right cornea was severely edematous, but the angle was gonioscopically unremarkable. The left eye demonstrated posterior embryotoxon and gonioscopy showed a white membrane in the peripheral iris extending through Schwalbe's line. The angle was open without evidence of inflammation. The patient underwent trabeculectomy of the right eye 114 days after birth, which resulted in normalization of the ocular pressure $(15 \mathrm{mmHg})$ and significant reduction of the corneal edema, tearing, and photophobia. Examination indicated mild atrophy of the iris in the right eye and retinal lesions bilaterally unchanged from the examination performed at birth. Real-time reverse transcriptase polymerase

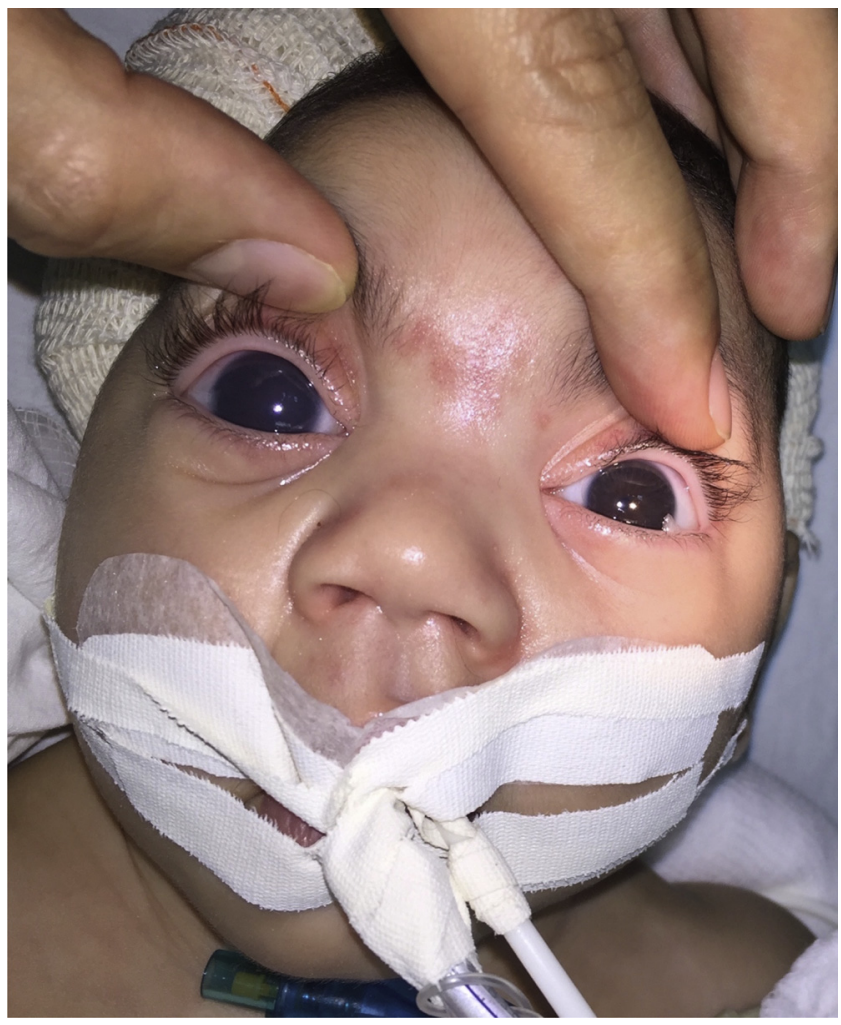

Figure 1. Severe corneal edema (blue-eye) associated with an enlarged right eye (buphthalmos) typically associated with congenital glaucoma.

chain reaction did not detect ZIKV-specific RNA in samples of the aqueous humor and vitreous obtained during surgery.

To our knowledge, this is the first report that describes glaucoma as a manifestation of congenital ZIKV infection. Clinicians should be aware of the possibility given the morbidity associated with glaucoma among infants. Furthermore, this case suggests that ZIKV may mediate damage to the anterior segment in addition to the posterior chamber of the eye. We reported a case of an infant with congenital ZIKV infection who presented with iris coloboma and lens subluxation at birth. ${ }^{1}$ Recently, uveitis has been reported as a manifestation of acute ZIKV infection in adults. ${ }^{5}$ With an infection occurring many months before birth, we did not detect viral RNA in the aqueous humor; however, further study is needed to determine whether glaucoma and anterior segment lesions are owing to the direct or indirect effects of the virus during gestation or postpartum, as well as the risk that these sequelae pose for newborn infants with congenital ZIKV infection. 


\section{Ophthalmology Volume a, Number a, Month 2016}

Acknowledgments. The authors are grateful to Dr Pedro F.C. Vasconcelos and Dr Sueli Rodrigues from Evandro Chagas Institute for assistance with ZIKV IgM ELISA assays.

Bruno de Paula Freitas, MD ${ }^{1,2}$

Albert I. Ko, MD 3,4

RicARDo KHOURI, PHD 3

Monica Mayoral, MD

Daniele Freitas Henriques, PhD ${ }^{5}$

Maurício Maia, MD, PhD ${ }^{2}$

Rubens Belfort, JR., MD, PhD ${ }^{2}$

${ }^{1}$ Department of Ophthalmology, Hospital Geral Roberto Santos, Salvador, Brazil; ${ }^{2}$ Vision Institute, Department of Ophthalmology, Paulista Medical School, Federal University of São Paulo, São Paulo, Brazil; ${ }^{3}$ Gonçalo Moniz Research Center, Oswaldo Cruz Foundation, Salvador, Brazil; ${ }^{4}$ Department of Epidemiology of Microbial Diseases, Yale School of Public Health, New Haven, Connecticut; ${ }^{5}$ Department of Arbovirology and Hemorrhagic Fevers, Evandro Chagas Institute, Ministry of Health, Ananindeua, Brazil

Financial Disclosures: The authors have no proprietary or commercial interest in any materials discussed in this article.

Author Contributions:

Conception and design: de Paula Freitas, Belfort

Analysis and interpretation: de Paula Freitas, Khouri, Mayoral, Belfort
Data collection: de Paula Freitas, Ko, Khouri, Freitas, Belfort Obtained funding: Not applicable

Overall responsibility: de Paula Freitas, Ko, Mayoral, Maia, Belfort

Correspondence:

Rubens Belfort Jr., MD, PhD, Department of Ophthalmology, Federal University of São Paulo, Paulista Medical School, Rua Botucatu, 821, São Paulo, SP 04023-062, Brazil. E-mail: prof.belfort@clinicabelfort. com.br.

\section{References}

1. de Paula Freitas B, de Oliveira Dias JR, Prazeres J, et al. Ocular findings in infants with microcephaly associated with presumed Zika virus congenital infection in Salvador, Brazil. JAMA Ophthalmol. 2016;134:529-535.

2. Ventura CV, Maia M, Bravo-Filho V, et al. Zika virus in Brazil and macular atrophy in a child with microcephaly. Lancet. 2016:387:228.

3. Ventura CV, Maia M, Ventura BV, et al. Ophthalmologic findings in infants with microcephaly and presumable intrauterus Zika virus infection. Arq Bras Oftalmol. 2016;79:1-3.

4. Petersen LR, Jamieson DJ, Powers AM, et al. Zika virus. N Engl J Med. 2016;374:1552-1563.

5. Furtado JM, Espósito DL, Klein TM, et al. Uveitis associated with Zika virus infection. N Engl J Med. 2016;375:394-396. 